\title{
COMPARING RELATIVISTIC AND NEWTONIAN DYNAMICS IN FIRST-ORDER LOGIC
}

\author{
JUDIT X. MADARÁSZ AND GERGELY SZÉKELY
}

\section{INTRODUCTION}

In this paper we introduce and compare Newtonian and relativistic dynamics as two theories of first-order logic (FOL). To illustrate the similarities between Newtonian and relativistic dynamics, we axiomatize them such that they differ in one axiom only. This one axiom difference, however, leads to radical differences in the predictions of the two theories. One of their major differences manifests itself in the relation between relativistic and rest masses, see Thms. 4.2 and 4.3.

The statement that the center-lines of a system of point masses viewed from two different reference frames are related exactly by the coordinate transformation between them seems to be a natural and harmless assumption; and it is natural and harmless in Newtonian dynamics, see Cor.4.8. However, in relativistic dynamics it leads to a contradiction, see Thm.4.1. Showing this surprising fact, which also illustrates the great difference between the two theories, is the main result of this paper.

Our work is directly related to Hilbert's 6th problem on axiomatization of physics. Moreover, it goes beyond this program since our general aim is not only to axiomatize physical theories but to investigate the relationship between the basic assumptions (axioms) and

Research supported by Hungarian National Foundation for Scientific Research grant No T73601 as well as by Bolyai Grant for Judit X. Madarász. 
the predictions (theorems) of the theories and to compare the axiom systems of related theories. Our another general aim is to provide a foundation of physics similar to that of mathematics.

For good reasons, the foundation of mathematics was performed strictly within FOL. One of these reasons is that staying within FOL helps to avoid tacit assumptions. Another reason is that FOL has a complete inference system while second-order logic (and thus any higher-order logic) cannot have one, see, e.g., [11, §IX. 1.6]. For further reasons for staying within FOL, see, e.g., [5, §Why FOL?], [7], [18, $\S 11],[19],[20]$.

There are many FOL axiomatizations of relativistic kinematics both special and general, see, e.g., [7], [8], [9], [12], [17]. However, as far as we know, our co-authored paper [6] is the only one which deals with the FOL axiomatization of relativistic dynamics, too. Newtonian and relativistic kinematics are compared in the level of axioms in $[5, \S 4.1]$. The main aim of this paper is to compare the key axioms and theorems of Newtonian and relativistic dynamics, too.

\section{A FIRST-ORDER LOGIC FRAME FOR DYNAMICS}

Our choice of vocabulary (basic concepts) is explained as follows. We represent motion as the changing of spatial location of bodies in time. To do so, we have reference-frames for coordinatizing events (sets of bodies) and, for simplicity, we associate reference-frames with observers. There are special kind of bodies which we call photons. For coordinatizing events, we use an ordered field in place of the field of real numbers. ${ }^{1}$ Thus the elements of this field are the quantities which we use for marking time and space. In our axioms of dynamics we use relativistic masses of bodies as a basic concept. 
Motivated by the above, we now turn to fixing the FOL language of our axiom systems. First we fix a natural number $d \geq 2$ for the dimension of spacetime. Our language contains the following non-logical symbols:

- unary relation symbols IOb (inertial observers), B (bodies), $\mathrm{Ph}$ (photons) and Q (quantities),

- binary function symbols + , • and a binary relation symbol $<$ (the field operations and the ordering on Q),

- a $2+d$-ary relation symbol W (world-view relation), and

- a binary function symbol M (mass function).

We translate $\operatorname{IOb}(x), \mathrm{B}(x), \mathrm{Ph}(x)$ and $\mathrm{Q}(x)$ into natural language as " $x$ is an (inertial) observer," " $x$ is a body," " $x$ is a photon," and " $x$ is a quantity." (A more careful wording would be " $x$ is a possible observer," " $x$ is a possible body," etc.) The bodies play the role of the "main characters" of our spacetime models and they are "observed" (coordinatized using the quantities) by the observers. This observation is coded by the world-view relation by translating $\mathrm{W}\left(x, y, z_{1}, \ldots, z_{d}\right)$ as "observer $x$ coordinatizes body $y$ at spacetime location $\left\langle z_{1}, \ldots, z_{d}\right\rangle$," (i.e., at space location $\left\langle z_{2}, \ldots, z_{d}\right\rangle$ at instant $z_{1}$ ). Finally we use the mass function to speak about the relativistic masses of bodies according to observers, i.e., " $\mathrm{M}(x, y)$ is the relativistic mass of body $y$ according to observer $x . "$

$\mathrm{IOb}(x), \mathrm{B}(x), \mathrm{Ph}(x), \mathrm{Q}(x), \mathrm{W}\left(x, y, z_{1}, \ldots, z_{d}\right), x=y$ and $x<y$ are the atomic formulas, where $x, y, z_{1}, \ldots, z_{d}$ can be arbitrary variables or terms built up from variables by using the field-operations and the mass function $\mathrm{M}$. The formulas are built up from these atomic formulas by using the logical connectives not $(\neg)$, and $(\Lambda)$, or $(\vee)$, implies $(\rightarrow)$, if-and-only-if $(\leftrightarrow)$ and the quantifiers exists $x(\exists x)$ and for all $x(\forall x)$ for every variable $x$. 
The models of this language are of the form

$$
\langle U ; \mathrm{IOb}, \mathrm{B}, \mathrm{Ph}, \mathrm{Q},+, \cdot,<, \mathrm{W}, \mathrm{M}\rangle,
$$

where $U$ is a nonempty set and $\mathrm{IOb}, \mathrm{B}, \mathrm{Ph}$ and $\mathrm{Q}$ are unary relations on $U$, etc. For simplicity we write $k \in \mathrm{IOb}$ in place of $\operatorname{IOb}(k)$, etc.

We use the notation $\mathrm{Q}^{n}:=\mathrm{Q} \times \ldots \times \mathrm{Q}(n$-times $)$ for the set of all $n$-tuples of elements of $\mathrm{Q}$. If $p \in \mathrm{Q}^{n}$, then we assume that $p=$ $\left\langle p_{1}, \ldots, p_{n}\right\rangle$, i.e., $p_{i} \in \mathrm{Q}$ denotes the $i$-th component of the $n$-tuple $p$. We write $\mathrm{W}(k, b, p)$ in place of $\mathrm{W}\left(k, b, p_{1}, \ldots, p_{d}\right)$, and we write $\forall p$ in place of $\forall p_{1}, \ldots, p_{d}$, etc.

We present each axiom at two levels. First we give an intuitive formulation, then we give a precise formalization using our logical notation (which can easily be translated into FOL formulas by inserting the definitions into the formalizations). We seek to formulate easily understandable axioms in FOL.

Our first axiom expresses very basic assumptions, such as: photons are bodies, etc.

AxFrame : $\mathrm{Ph} \subseteq \mathrm{B}$, the quantity part $\langle\mathrm{Q} ;+, \cdot,<\rangle$ is a Euclidean $^{2}$ ordered field, and the masses are positive elements of the quantity part, i.e., $\mathrm{Q}(\mathrm{M}(k, b)) \wedge \mathrm{M}(k, b)>0$.

For the FOL definition of linearly ordered field, see, e.g., [10]. We use the usual field operations $0,1,-, /, \sqrt{ }$ definable within FOL. We also use the vector-space structure of $\mathrm{Q}^{n}$, i.e., if $p, q \in \mathrm{Q}^{n}$ and $\lambda \in \mathrm{Q}$, then $p+q,-p, \lambda p \in \mathrm{Q}^{n}$. The Euclidean length of $p \in \mathrm{Q}^{n}$ is defined as $|p|:=\sqrt{p_{1}^{2}+\ldots+p_{n}^{2}}$, for any $n \geq 1$. The Euclidean distance of $p, q \in \mathrm{Q}^{n}$ is defined as $|p q|:=|p-q|$. As usual, $\ell$ is called a line iff there are $p, q \in \mathrm{Q}^{d}$ such that $q \neq\langle 0, \ldots, 0\rangle$ and $\ell=\{p+\lambda q: \lambda \in \mathrm{Q}\}$. And $\mathrm{Q}^{+}:=\{\lambda \in \mathrm{Q}: 0<\lambda\}$ denotes the set of positive elements of $\mathrm{Q}$. Set $\mathrm{Q}^{d}$ is called coordinate system and its elements are referred 
to as coordinate points. We use the notations

$$
p_{\sigma}:=\left\langle p_{2}, \ldots, p_{d}\right\rangle \text { and } p_{\tau}:=p_{1}
$$

for the space component and the time component of $p \in \mathrm{Q}^{d}$, respectively. The event $e v_{k}(p)$ is the set of bodies observed by observer $k$ at coordinate point $p$, i.e.,

$$
e v_{k}(p):=\{b \in \mathrm{B}: \mathrm{W}(k, b, p)\} .
$$

The world-line of body $b$ according to observer $k$ is defined as the set of coordinate points where $b$ was observed by $k$, i.e.,

$$
w l_{k}(b):=\left\{p \in \mathrm{Q}^{d}: W(k, b, p)\right\} .
$$

\section{Kinematics}

In this section we formulate our axioms on kinematics. Our first axiom on observers states that they see the same events.

AxEv : All observers coordinatize the very same events:

$$
\forall k, h \in \mathrm{IOb} \forall p \in \mathrm{Q}^{d} \exists q \in \mathrm{Q}^{d} \quad e v_{k}(p)=e v_{h}(q)
$$

To introduce our next axiom, we need a concept of inertial bodies. A body is called inertial iff its world-line is a line for every observer. The set of inertial bodies is denoted by IB, i.e.,

$$
\mathrm{IB}:=\left\{b \in \mathrm{B}: \forall k \in \mathrm{IOb} w l_{k}(b) \text { is a line }\right\} .
$$

AxThEx below states that each observer can make thought experiments in which it assumes the existence of "slowly moving" inertial bodies (see, e.g., [4, p.622]):

AxThEx : For each observer there is a positive speed limit such that in each spacetime location, in each direction, with any 
speed less than this limit it is possible to "send out" an inertial body:

$$
\begin{aligned}
& \forall k \in \mathrm{IOb} \exists \lambda \in \mathrm{Q}^{+} \forall p, q \in \mathrm{Q}^{d} \exists b \in \mathrm{IB} \\
& \qquad\left(\left|(p-q)_{\sigma}\right|<\lambda(p-q)_{\tau} \rightarrow p, q \in w l_{k}(b)\right) .
\end{aligned}
$$

The following axiom system will be the common core of our axiom systems for relativistic and Newtonian kinematics:

$$
\text { Kin }:=\{A x E v, A x T h E x, \text { AxFrame }\} .
$$

The world-view transformation between the coordinate systems of observers $k$ and $h$ is the set of pairs of coordinate points $\langle p, q\rangle$ such that $k$ and $h$ observe the same event in $p$ and $q$, respectively, i.e.,

$$
w_{k h}:=\left\{\langle p, q\rangle \in \mathrm{Q}^{d} \times \mathrm{Q}^{d}: e v_{k}(p)=e v_{h}(q)\right\} .
$$

If $R$ is a binary relation and $X$ is a set, $R[X]$ denotes the $R$-image of $X$, i.e., $R[X]:=\{b: \exists a \in X\langle a, b\rangle \in R\}$.

Proposition 3.1. Assuming Kin, the world-view transformations are bijections and take lines to lines, i.e., $w_{k h}[\ell]$ is a line for every line $\ell$ and $k, h \in \mathrm{IOb}$.

A proof can be obtained from that of Thm.3.1.1 in [5, pp.160-170].

We extend Kin to an axiom system for special relativity by assuming that the speed of light is 1 according to any observer.

$\mathrm{AxPh}$ : The world-lines of photons are of slope 1, and for every observer, there is a photon through two coordinate points if their slope is 1 :

$$
\begin{aligned}
\forall k \in \mathrm{IOb} \forall p, q \in \mathrm{Q}^{d} \quad\left(\left|p_{\sigma}-q_{\sigma}\right|=\left|p_{\tau}-q_{\tau}\right| \leftrightarrow\right. & \\
& \left.\exists p h \in \mathrm{Ph} \quad p, q \in w l_{k}(p h)\right) .
\end{aligned}
$$


We axiomatize special relativistic kinematics as follows:

$$
\text { SpecRelKin }:=\operatorname{Kin} \cup\{\mathrm{AxPh}\} \text {. }
$$

Convention 3.2. Whenever we write " $w_{k h}(p)$," we mean that there is a unique $q \in \mathrm{Q}^{d}$ such that $\langle p, q\rangle \in w_{k h}$, and $w_{k h}(p)$ denotes this $q$.

Convention 3.3. We use the equation sign "=" in the sense of existential equality (of partial algebra theory [1]), i.e., $\alpha=\beta$ abbreviates that both $\alpha$ and $\beta$ are defined and they are equal. See [13, Conv.2.3.10, p.31] and [5, Conv.2.3.10, p.61]. Similar convention applies for the binary relations " $<$ " and " $\neq$."

To get an axiom system for Newtonian kinematics, we extend Kin by an axiom saying that the simultaneity of events is independent from observers.

AxAbsSim : Simultaneity is absolute, i.e.,

$$
\forall k, h \in \mathrm{IOb} \forall p, q \in \mathrm{Q}^{d}\left(p_{\tau}=q_{\tau} \rightarrow w_{k h}(p)_{\tau}=w_{k h}(q)_{\tau}\right) .
$$

We axiomatize Newtonian kinematics as follows:

$$
\text { NewtKin }:=\text { Kin } \cup\{\text { AxAbsSim }\} \text {. }
$$

Let us note that SpecRelKin and NewtKin differ in one axiom only. But we will see in Prop.3.4 below that these two axiom systems are very different, e.g., they are inconsistent together if we assume that there are observers moving relative to each other. To formulate this statement we need the following definition.

The speed $v_{k}(b)$ of body $b$ according to observer $k$ is defined as:

$$
v_{k}(b):=\frac{\left|p_{\sigma}-q_{\sigma}\right|}{\left|p_{\tau}-q_{\tau}\right|}, \text { for } p, q \in w l_{k}(b) \text { with } p_{\tau} \neq q_{\tau}
$$

if $w l_{k}(b)$ is a subset of a line and contains coordinate points $p$ and $q$ with $p_{\tau} \neq q_{\tau}$, otherwise $v_{k}(b)$ is undefined. 
Ax $\exists \mathrm{IOb}$ : There are observers moving relative to each other.

$$
\exists k, h \in \mathrm{IOb} \exists b \in \mathrm{IB} \quad v_{h}(b) \neq v_{k}(b)=0 .
$$

Proposition 3.4. SpecRelKin $\cup$ NewtKin $\cup\{A x \exists I O b\}$ is inconsistent.

This proposition is a corollary of Thm.3.6 below.

While in Newtonian kinematics there is no speed limit for observers SpecRelKin implies that no observer can move faster than light if $d \geq 3$ by the following theorem.

Theorem 3.5. Assume $d \geq 3$ and SpecRelKin. Then there are no faster than light observers, i.e.,

$$
\forall k, h \in \mathrm{IOb} \forall b \in \mathrm{B}\left(v_{k}(b)=0 \rightarrow v_{h}(b)<1\right) .
$$

Moreover, $\forall k, h \in \mathrm{IOb} \forall b \in \mathrm{B}\left(v_{k}(b)<1 \rightarrow v_{h}(b)<1\right)$.

In the first formula of the theorem, the speeds of observers are captured by speaking about resting bodies. For proof, see, e.g., [2, Prop.1, Thm.3], [13, 2.3.5, 2.8.25, 3.2.13], [16, Thm.3, Thm.5]. We note that the theorem remains true if we omit AxThEx from SpecRelKin.

Lines $\ell$ and $\ell^{\prime}$ are said to be orthogonal in the Euclidean sense iff there are $p, p^{\prime} \in \ell$ and $q, q^{\prime} \in \ell^{\prime}$ such that $p \neq p^{\prime}, q \neq q^{\prime}$, and

$$
\left(p_{1}-p_{1}^{\prime}\right)\left(q_{1}-q_{1}^{\prime}\right)+\left(p_{2}-p_{2}^{\prime}\right)\left(q_{2}-q_{2}^{\prime}\right)+\ldots+\left(p_{d}-p_{d}^{\prime}\right)\left(q_{d}-q_{d}^{\prime}\right)=0 .
$$

If $p, q \in \mathrm{Q}^{d}$ and $p \neq q$, then $p q$ denotes the line passing through coordinate points $p$ and $q$.

By Thm.3.6, two clocks separated in direction not orthogonal to the direction of movement get out of synchronism. 
Theorem 3.6. Assume SpecRelKin. Then two clocks remain in synchronism iff they are separated in direction orthogonal to the direction of movement. Formally: Let $k, h \in \mathrm{IOb}, b \in \mathrm{IB}$ and $p, q \in \mathrm{Q}^{d}$ be such that $v_{k}(b)=0, p \neq q$ and $p_{\tau}=q_{\tau}$. Then $w_{h k}(p)_{\tau}=w_{h k}(q)_{\tau}$ iff $p q$ is orthogonal to $w l_{h}(b)$ in the Euclidean sense.

For proof, see, e.g., [4, Thm.11.4, p.626].

To formulate one more theorem on SpecRelKin, we need the following definitions: Let $p, q, r, s \in \mathrm{Q}^{d}$. The Minkowski length of $p$ is

$$
\mu(p):=\left\{\begin{aligned}
\sqrt{\left.\left|p_{\tau}^{2}-\right| p_{\sigma}\right|^{2} \mid} & \text { if } p_{\tau}^{2}-\left|p_{\sigma}\right|^{2} \geq 0 \\
-\sqrt{\left.\left|p_{\tau}^{2}-\right| p_{\sigma}\right|^{2} \mid} & \text { otherwise }
\end{aligned}\right.
$$

and the Minkowski distance of $p$ and $q$ is $\mu(p, q):=\mu(p-q)$. Segments $[p q]$ and $[r s]$ are called Minkowski equidistant iff $\mu(p, q)=$ $\mu(r, s)$.

Theorem 3.7. Assume SpecRelKin. Then the world-view transformations preserve the Minkowski equidistance, i.e.,

$$
\begin{aligned}
& \forall k, h \in \mathrm{IOb} \forall p, q, r, s \in \mathrm{Q}^{d} \\
& \quad\left(\mu(p, q)=\mu(r, s) \rightarrow \mu\left(w_{k h}(p), w_{k h}(q)\right)=\mu\left(w_{k h}(r), w_{k h}(s)\right)\right) .
\end{aligned}
$$

Idea of proof of Thm.3.7 is in $\S 5$.

\section{Dynamics}

In this section we formulate our axioms on dynamics. For convenience we use the notation $m_{k}(b):=M(k, b)$ for the relativistic mass of body $b$ according to observer $k$.

The spacetime location $\operatorname{loc}_{k}(b, t)$ of body $b$ at time instance $t \in \mathrm{Q}$ according to observer $k$ is defined to be the coordinate point $p$ for which 
$p \in w l_{k}(b)$ and $p_{\tau}=t$ if there is such a unique $p$, otherwise $\operatorname{loc}_{k}(b, t)$ is undefined, see Fig.1.

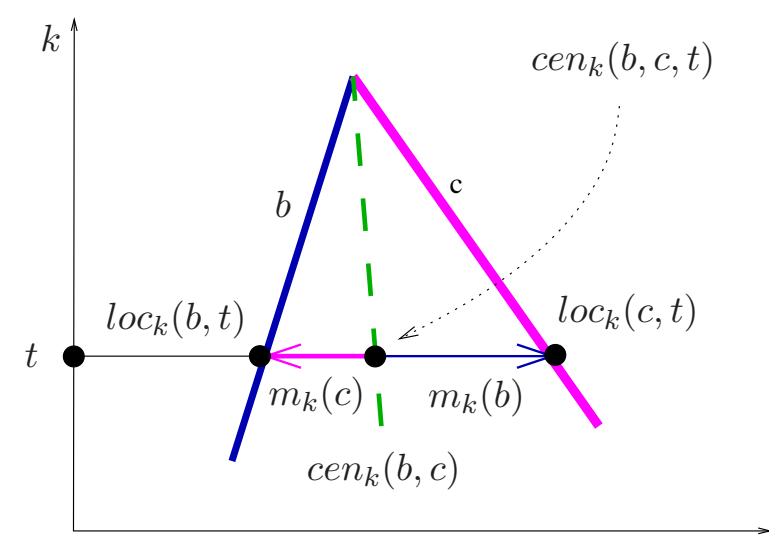

Figure 1. Illustration of $l o c_{k}(b, t), c e n_{k}(b, c, t)$ and $c e n_{k}(b, c)$.

The center of the masses $c e n_{k}(b, c, t)$ of bodies $b$ and $c$ at time instance $t$ according to observer $k$ is defined to be the coordinate point $q$ such that $q_{\tau}=t$ and $q$ is the point on the line-segment between $\operatorname{loc}_{k}(b, t)$ and $l o c_{k}(c, t)$ whose distances from these two end-points have the same proportion as that of the relativistic masses of $b$ and $c$; and it is closer to the "more massive" body, i.e.:

$$
m_{k}(b)\left(\operatorname{loc}_{k}(b, t)-\operatorname{cen}_{k}(b, c, t)\right)=m_{k}(c)\left(c e n_{k}(b, c, t)-\operatorname{loc}_{k}(c, t)\right)
$$

if $\operatorname{loc}_{k}(b, t)$ and $l o c_{k}(c, t)$ are defined, and otherwise $c e n_{k}(b, c, t)$ is undefined, see Fig.1. We note that an explicit definition for $\operatorname{cen}_{k}(b, c, t)$ is the following:

$$
c e n_{k}(b, c, t)=\frac{m_{k}(b)}{m_{k}(b)+m_{k}(c)} \operatorname{loc}_{k}(b, t)+\frac{m_{k}(c)}{m_{k}(b)+m_{k}(c)} \operatorname{loc}_{k}(c, t),
$$

if $l o c_{k}(b, t)$ and $l o c_{k}(c, t)$ are defined, otherwise $c e n_{k}(b, c, t)$ is undefined. The center-line of the masses of bodies $b$ and $c$ according to observer 
$k$ is defined as

$$
\operatorname{cen}_{k}(b, c):=\left\{c e n_{k}(b, c, t): t \in \mathrm{Q} \text { and } \operatorname{cen}_{k}(b, c, t) \text { is defined }\right\} .
$$

In Newtonian dynamics two bodies can be substituted by one body living on the center-line of the two bodies and having mass equal to the sum of the masses of the two bodies. The crucial point in this statement is that different observers agree as for the center-line of inertial bodies (up to world-view transformations), which can be formalized as follows.

AxCen : The world-view transformations take the center-line of two inertial bodies to the center-line of the two bodies.

$$
\forall k, h \in \mathrm{IOb} \forall b, c \in \mathrm{IB} \quad w_{k h}\left[\operatorname{cen}_{k}(b, c)\right]=\operatorname{cen}_{h}(b, c) .
$$

However intuitive and natural AxCen is, it does not hold in the "standard model" of special relativity. Moreover, it is inconsistent with SpecRelKin if we assume that there are observers moving relative to each other.

Theorem 4.1. Assume $d \geq 3$. Then SpecRelKin $\cup\{A x \exists I O b, A x C e n\}$ is inconsistent.

The proof of Thm.4.1 is in section 5 .

Thus, by Thm.4.1, two bodies cannot be replaced by one in relativistic dynamics. Therefore, if we want to build a consistent relativistic dynamics based on this assumption, we have to weaken AxCen. The solution is to assume it only for meeting bodies.

AxCen ${ }^{-}$: The world-view transformations take the center-line of two meeting inertial bodies to the center-line of the two bodies.

$$
\begin{aligned}
& \forall k, h \in \mathrm{IOb} \forall b, c \in \mathrm{IB} \\
& \quad\left(w l_{k}(b) \cap w l_{k}(c) \neq \emptyset \rightarrow w_{k h}\left[\operatorname{cen}_{k}(b, c)\right]=\operatorname{cen}_{h}(b, c)\right) .
\end{aligned}
$$


The main axiom of dynamics is $\mathrm{AxCen}^{-}$. The remaining axioms of our axiom system are only simplifying axioms.

The rest mass $m_{0}(b)$ of body $b$ is defined as $m_{0}(b)=\lambda$ if $(1)$ there is an observer according to which $b$ is at rest and the relativistic mass of $b$ is $\lambda$, and (2) for every observer according to which $b$ is at rest the relativistic mass of $b$ is $\lambda$, i.e., $m_{0}(b)=\lambda$ iff $\exists k \in \operatorname{IOb}\left(v_{k}(b)=0 \wedge m_{k}(b)=\lambda\right) \wedge \forall k \in \operatorname{IOb}\left(v_{k}(b)=0 \rightarrow m_{k}(b)=\lambda\right)$.

AxCen- (together with SpecRelKin) implies that the mass of a body necessarily depends on the observer. The reason for this fact is that the simultaneities of observers moving relative to each other in SpecRelKin differ from each other, and this implies that the proportions involved in $\mathrm{AxCen}{ }^{-}$change, too. See Prop.4.1 and Fig.3 in [6]. The next axiom states that the relativistic mass of a body depends at most on its rest mass and its speed.

AxSpeed: The relativistic masses of two inertial bodies are the same if both of their rest masses and speeds are equal:

$\forall k \in \mathrm{IOb} \forall b, c \in \mathrm{IB}$

$$
\left(\left(m_{0}(b)=m_{0}(c) \wedge v_{k}(b)=v_{k}(c)\right) \rightarrow m_{k}(b)=m_{k}(c)\right) .
$$

Our next axiom on dynamics states that each observer can make experiments by putting stationary inertial bodies with arbitrary rest mass to any coordinate point.

AxRest : In the coordinate system of any observer there is a resting inertial body with arbitrary rest mass at any coordinate point.

$$
\forall k \in \mathrm{IOb} \forall \lambda \in \mathrm{Q}^{+} \forall p \in \mathrm{Q}^{d} \exists b \in \mathrm{IB}\left(m_{0}(b)=\lambda \wedge p \in w l_{k}(b)\right) .
$$

Let $\mathrm{IB}_{0}$ denote the set of inertial bodies having rest mass. 
AxMedian : For every two inertial bodies having rest mass, there is an observer for which they have the same speed:

$$
\forall b, c \in \mathrm{IB}_{0} \exists k \in \mathrm{IOb} \quad v_{k}(b)=v_{k}(c) .
$$

Let us collect the axioms for dynamics together.

$$
\text { Dyn }:=\left\{\mathrm{AxCen}^{-}, \mathrm{AxSpeed}, \text { AxRest, AxMedian }\right\} .
$$

By adding Dyn to our kinematical axiom systems we get the respective dynamical ones.

$$
\begin{aligned}
\text { NewtDyn } & :=\text { Dyn } \cup \text { Kin } \cup\{\text { AxAbsTime }\}=\text { Dyn } \cup \text { NewtKin, } \\
\text { SpecRelDyn } & :=\text { Dyn } \cup \text { Kin } \cup\{A x P h\}=\text { Dyn } \cup \text { SpecRelKin. }
\end{aligned}
$$

Let us note that Dyn is the common dynamical core of the two axiom systems, which also differ in one axiom only.

Thms. 4.2 and 4.3 below give the connection between the rest mass and the relativistic mass of an inertial body. Their conclusions are well known results of relativistic and Newtonian dynamics. However, in the usual literature, the assumptions are stronger and not stated explicitly.

Theorem 4.2. Assume SpecRelDyn. Let $k$ be an observer and $b$ be an inertial body having rest mass. Then $v_{k}(b)<1$ and

$$
m_{0}(b)=m_{k}(b) \sqrt{1-v_{k}(b)^{2}} .
$$

Theorem 4.3. Assume NewtDyn. Let $k$ be an observer and $b$ be an inertial body having rest mass. Then

$$
m_{0}(b)=m_{k}(b) .
$$

The proofs of Thms. 4.2 and 4.3 are in section 5 .

Let us note that, by Thms. 4.2 and 4.3, axiom systems SpecRelDyn and NewtDyn differing in one axiom have radically different consequences. 
Coordinate points $p, q$ and $r$ are called collinear iff there is a line $\ell$ such that $p, q, r \in \ell$. A map $f: \mathrm{Q}^{d} \longrightarrow \mathrm{Q}^{d}$ is called an affine transformation, if it preserves collinearity and the ratios of distances, i.e., for every distinct and collinear coordinate points $p, q$ and $r$ coordinate points $f(p), f(q)$ and $f(r)$ are collinear and $|p q| \cdot|f(q) f(r)|=$ $|f(p) f(q)| \cdot|q r|$.

By Prop.3.1, it follows that the world-view transformations are affine transformations composed by filed-automorphism induced mappings in models of Kin. It can be proved that, in models of SpecRelKin and NewtKin, the world-view transformations are not necessarily affine transformations, i.e., there are models in which field-automorphism induced mappings occur in the world-view transformations. By Thm.4.4, this is not the case in models of NewtDyn and SpecRelDyn.

Theorem 4.4. The world-view transformations are bijective affine transformations in models of NewtDyn and SpecRelDyn.

The proof of Thm.4.4 is in section 5 .

By Thm.4.2, SpecRelDyn implies that for every inertial body $b$ the quantity $m_{k}(b) \sqrt{1-v_{k}(b)^{2}}$ is independent of observer $k$ if $b$ has rest mass. By Thm.4.5, the same holds for every inertial body $b$ moving slower than light.

Theorem 4.5. Assume SpecRelDyn. Let $b$ be an inertial body such that $v_{k}(b)<1$ for an observer $k$. Then $v_{k}(b)<1$ for every observer $k$ and

$$
\forall k, h \in \mathrm{IOb} \quad m_{k}(b) \sqrt{1-v_{k}(b)^{2}}=m_{h}(b) \sqrt{1-v_{h}(b)^{2}} .
$$

The proof of Thm.4.5 is in section 5 .

By our definition, the rest mass of an inertial body can be undefined even if there is an observer according to which the body is at rest. By 
the following immediate corollary of Thm.4.5, the rest mass of a body is defined whenever there is an observer according to which the body is at rest if we assume SpecRelDyn.

Corollary 4.6. Assume SpecRelDyn. Let $b$ be an inertial body such that $v_{k}(b)=0$ for some $k \in \mathrm{IOb}$. Then $b$ has a rest mass.

By Thm.4.3, axiom system NewtDyn implies that relativistic mass of an inertial body is observer independent if the body has rest mass. By Thm.4.7, the same holds for inertial bodies with "finite" speeds.

Theorem 4.7. Assume NewtDyn. Let $b$ be an inertial body such that $v_{k}(b)$ is defined for some $k \in \mathrm{IOb}$. Then

$$
\forall k, h \in \mathrm{IOb} \quad m_{k}(b)=m_{h}(b)
$$

The proof of Thm.4.7 is in section 5 .

By the following corollary, theory NewtDyn implies AxCen, the axiom which is inconsistent with SpecReIDyn, see Thm.4.1. This fact also shows great difference between the two theories of dynamics.

Corollary 4.8. NewtDyn $\models$ AxCen.

The proof of Cor. 4.8 is in section 5 .

\section{ProOfS}

Idea of proof of Thm.3.7. Assume first that $d>2$. One can prove, by Alexandrov-Zeeman theorem, that every world-view transformation is a composition of a Poincaré transformation, a dilation and a field-automorphism-induced mapping, cf. [3, Thm.1.2]. All of these mappings preserve the Minkowski equidistance. Thus the world-view transformations also preserve the Minkowski equidistance. 
We note that a similar proof can be obtained for $d=2$, cf. [3, Thm.1.4].

Another proof can be obtained as follows. Coordinate points $p$ and $q$ are called lightlike separated, in symbols $p \lambda q$ iff $\left|p_{\tau}-q_{\tau}\right|=\left|p_{\sigma}-q_{\sigma}\right|$. Furthermore, $p$ and $q$ are timelike separated iff $\left|p_{\tau}-q_{\tau}\right|>\left|p_{\sigma}-q_{\sigma}\right|$. Assume first that $d=2$. Let $p, q$ and $q^{\prime}$ be distinct coordinate points. It can be seen that

$$
\begin{aligned}
\mu(p, q)= & \mu\left(p, q^{\prime}\right) \leftrightarrow \\
& \exists s, s^{\prime}\left(s \neq s^{\prime} \wedge q \lambda s \wedge q^{\prime} \lambda s \wedge q \lambda s^{\prime} \wedge q^{\prime} \lambda s^{\prime} \wedge \operatorname{coll}\left(p, s, s^{\prime}\right)\right) .
\end{aligned}
$$

Then timelike separatedness is FOL definable from lightlike separatedness by Alexandrov-Zeeman theorem. Let $p, q$ and $q^{\prime}$ be distinct points such that $p$ and $q$ are timelike separated and the same holds for $p$ and $q^{\prime}$. Then one can see that (1) above holds for $p, q$ and $q^{\prime}$.

By the above one can prove that Minkowski equidistance for timelike separated pairs of points is FOL definable from lightlike separatedness and collinearity. World-view transformations preserve lightlike separatedness and collinearity by AxPh and Prop.3.1. Thus they preserve Minkowski equidistance for timelike separated pairs of points. The general case can be reduced to the timelike and lightlike cases.

Proof of Thm.4.1. The proof goes by contradiction. Let $\mathfrak{M}$ be a model of SpecRelKin $\cup\{\mathrm{A} x \exists \mathrm{IOb}, \mathrm{A} \times \mathrm{Cen}\}$. Let $k, h \in \mathrm{IOb}$ and $b \in \mathrm{IB}$ be such that $v_{h}(b) \neq v_{k}(b)=0$, see Fig.2. Let $c \in$ IB be such that $v_{h}(c)=0$ and $w l_{h}(b)$ and $w l_{h}(c)$ do not meet, i.e., $w l_{h}(b)$ and $w l_{h}(c)$ are skew lines. Such $c$ exists by AxThEx. Furthermore, $v_{h}(b)<1$ and $v_{k}(c)<1$ by Thm.3.5. Thus $w l_{h}(b)$ and $w l_{k}(c)$ are not "horizontal" lines, i.e., for every $t \in \mathrm{Q}$ there are $p \in w l_{h}(b)$ and $q \in w l_{k}(c)$ such that $t=p_{\tau}=q_{\tau}$. Let $p \in w l_{h}(c)$ and $q \in w l_{h}(b)$ be such that $p_{\tau}=q_{\tau}$ and $p q$ is not 
orthogonal to $w l_{h}(b)$ in the Euclidean sense. It is easy to see that there are such $p$ and $q$. Then by Thm.3.6, $w_{h k}(p)_{\tau} \neq w_{h k}(q)_{\tau}$. Let $r \in w l_{h}(c)$ and $s \in w l_{h}(b)$ be such that $w_{h k}(q)_{\tau}=w_{h k}(r)_{\tau}$ and $r_{\tau}=s_{\tau}$. Then $p q$ and $r s$ are skew lines since the world-lines of $b$ and $c$ are skew lines. Center-line $c e n_{h}(b, c)$ intersects lines $p q$ and $r s$ and it does not go through points $p, q, r$ and $s$. Thus it does not intersect line $q r$ since the world-lines of $b$ and $c$ are skew lines. On the other hand center-line $c e n_{k}(b, c)$ intersects line $w_{h k}(q) w_{h k}(r)$. That is a contradiction since the world-view transformations are bijections taking lines to lines and center-lines to center-lines by Thm.3.1 and AxCen.

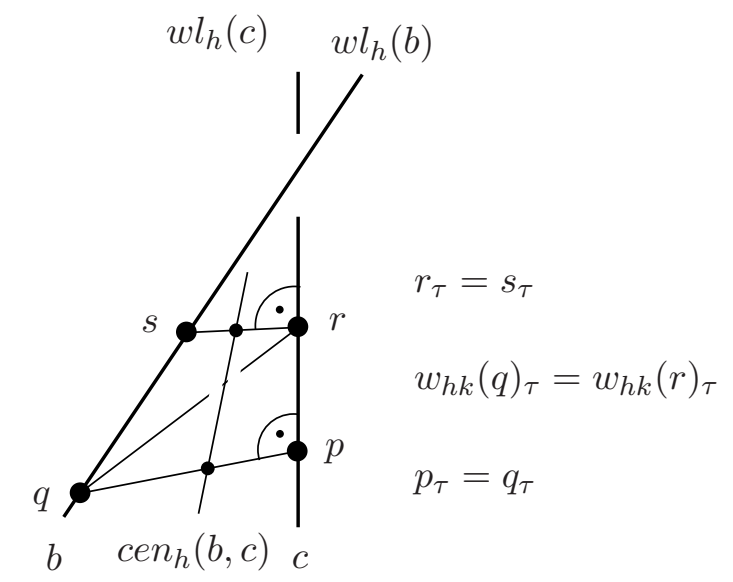

coordinate system of $h$

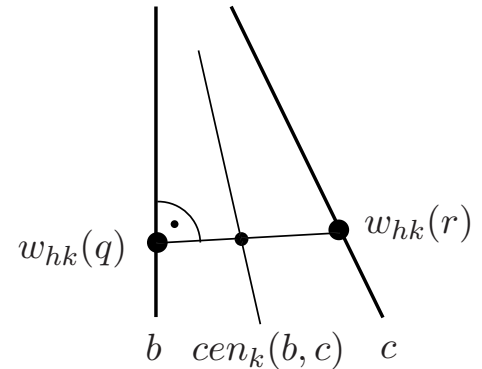

coordinate system of $k$

FiguRE 2. Illustration for the proof of Thm.4.1.

Proof of Thm.4.2. Let $k$ be an observer and $b$ be an inertial body having rest mass. Let $v:=v_{k}(b)$.

First we prove from SpecRelDyn that $v<1$. For $d \geq 3, v<1$ already follows from SpecRelKin by Thm.3.5. To see that $v<1$ for $d=2$, let 
$b^{\prime}$ be a body having rest mass such that $v_{k}\left(b^{\prime}\right)=0$. Such a $b^{\prime}$ exists by AxRest. By AxMedian there is an observer according to which $b$ and $b^{\prime}$ have the same speeds. But then, by SpecRelKin, it can be proved that $v<1$, cf. [5, Thm.2.7.2, p.110].

If $v=0$, the conclusion of the theorem holds. Thus we can assume that $v \neq 0$. Let $c$ be an inertial body such that $v_{k}(c)=0, m_{0}(c)=$ $m_{0}(b)$ and $b$ and $c$ meet. See Fig.3. Such a $c$ exists by AxRest. Let $A$ be

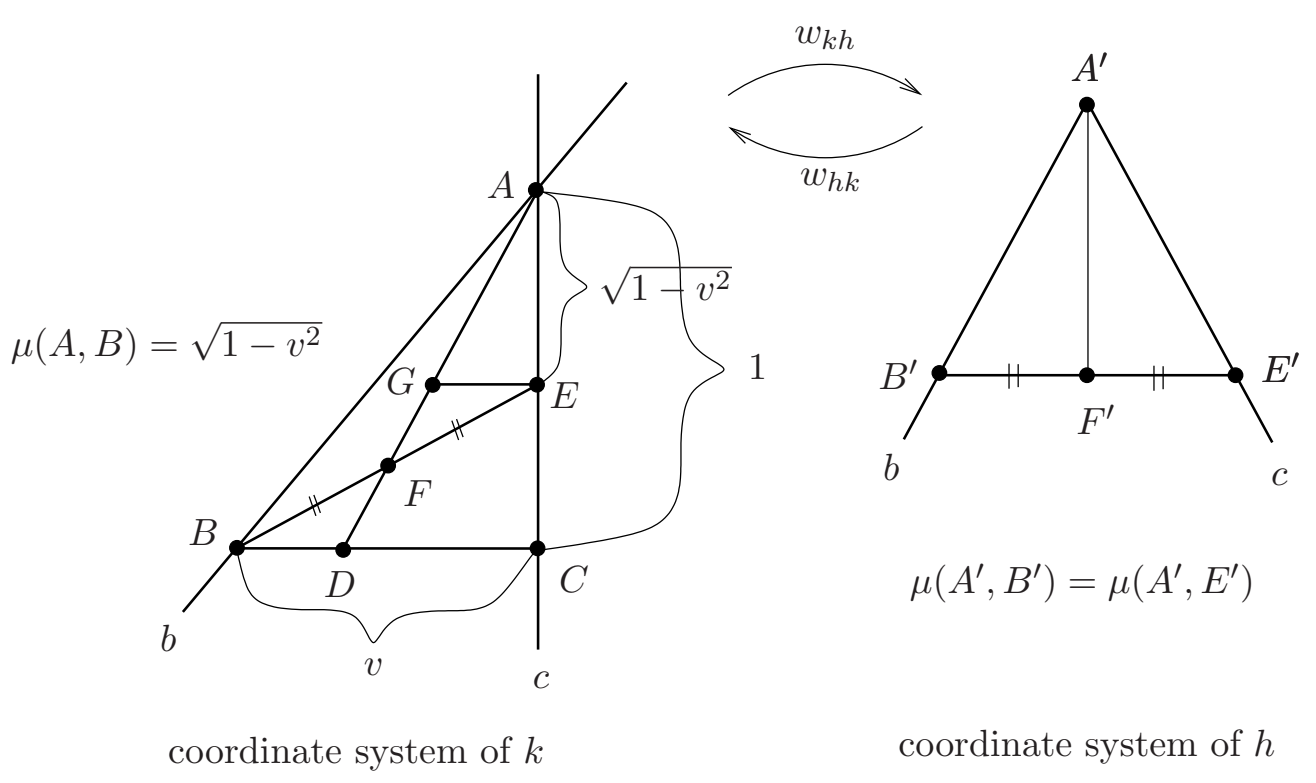

FiguRE 3. Illustration for the proof of Thm.4.2.

the coordinate point where the world-lines of $b$ and $c$ meet. Let $B$ and $C$ be the coordinate points on the world-lines of $b$ and $c$, respectively, such that $A_{\tau}-C_{\tau}=1$ and $B_{\tau}=C_{\tau}$. Then $|A C|=1,|B C|=v$ and $\mu(A, B)=\sqrt{1-v^{2}}$. Let $D$ be the center of masses of $b$ and $c$ at $B_{\tau}=C_{\tau}$, i.e., $D:=\operatorname{cen}_{k}\left(b, c, B_{\tau}\right)$. By definition of center of masses, 
$m_{k}(b)|B D|=m_{0}(b)|D C|$. Thus

$$
m_{0}(b)=\frac{|B D|}{|D C|} m_{k}(b) .
$$

Let $h$ be an observer according to which the speeds of $b$ and $c$ coincide. Such an $h$ exists by AxMedian. Let us turn our attention to the coordinate system of observer $h$ illustrated by the right hand side of Fig.3. Let $A^{\prime}$ and $B^{\prime}$ be the $w_{k h}$-images of $A$ and $B$, respectively. Let $E^{\prime}$ be a coordinate point on the world-line of $c$ such that $E_{\tau}^{\prime}=B_{\tau}^{\prime}$. Let $F^{\prime}$ be the center of masses of $b$ and $c$ at $E_{\tau}^{\prime}=B_{\tau}^{\prime}$, i.e., $F^{\prime}:=c e n_{h}\left(b, c, E_{\tau}^{\prime}\right)$. Since the rest masses and the speeds of $b$ and $c$ coincide, their relativistic masses coincide by AxSpeed. Thus $\left|B^{\prime} F^{\prime}\right|=\left|F^{\prime} E^{\prime}\right|$ by the definition of center of masses. Consequently, $\mu\left(A^{\prime}, B^{\prime}\right)=\mu\left(A^{\prime}, E^{\prime}\right)$, i.e., segments $\left[A^{\prime} B^{\prime}\right]$ and $\left[A^{\prime} E^{\prime}\right]$ are Minkowski equidistant.

Now we turn our attention to the coordinate system of observer $k$ illustrated by the left hand side of Fig.3. Let $F$ and $E$ be the $w_{h k}$ images of $F^{\prime}$ and $E^{\prime}$. Then, by $\mathrm{AxCen}^{-}, F \in A D=\operatorname{cen}_{k}(b, c)$ since $F^{\prime} \in c e n_{h}(b, c)$. Furthermore, $E \in B F$ since $w_{h k}$ takes lines to lines by Prop.3.1. The world-view transformation $w_{h k}$ preserve the Minkowski equidistance by Thm.3.7. Consequently, segments $[A B]$ and $[A E]$ as well as $[B F]$ and $[F E]$ are Minkowski equidistant. Thus $|A E|=\mu(A, E)=\mu(A, B)=\sqrt{1-v^{2}}$ and $|B F|=|F E|$.

Triangles $B D F$ and $E G F$ are congruent and triangles $A G E$ and $A D C$ are similar. Thus

$$
\frac{|B D|}{|D C|}=\frac{|G E|}{|D C|}=\frac{|A E|}{|A C|}=\sqrt{1-v^{2}} .
$$

By that and $(2)$, we get $m_{0}(b)=m_{k}(b) \sqrt{1-v^{2}}$. That completes the proof. 
Proof of Thm.4.3. Let $k$ be an observer and $b$ be an inertial body having rest mass. Then there is an observer $k^{\prime}$ such that $v_{k^{\prime}}(b)=0$. Thus, by AxAbsSim, $v_{k}(b)$ is not "infinite," i.e., $v_{k}(b)$ is defined. If $v_{k}(b)=0$, the conclusion of the theorem holds. Assume that $v_{k}(b) \neq 0$. Let $c$ be an inertial body such that $v_{k}(c)=0, m_{0}(c)=m_{0}(b)$ and $b$ and $c$ meet, see the left-hand side of Fig.4. Such a $c$ exists by AxRest. Let $h$

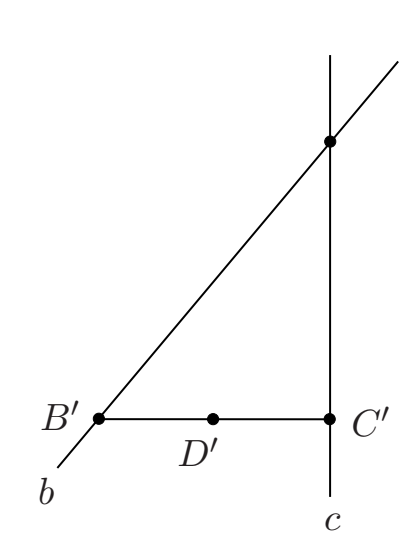

coordinate system of $k$

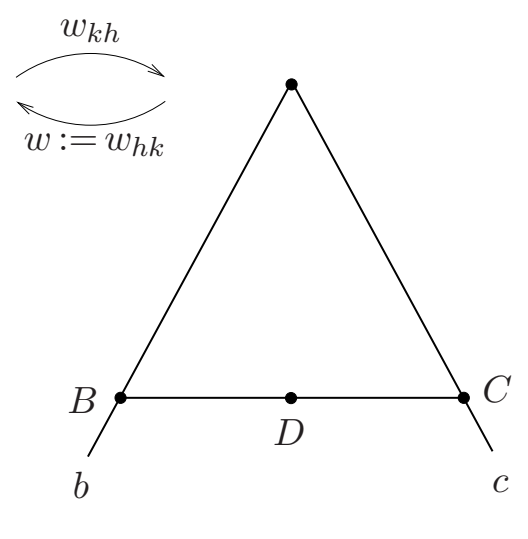

coordinate system of $h$

FiguRE 4. Illustration for the proof of Thm.4.3.

be an observer according to which the speeds of $b$ and $c$ coincide. Such an $h$ exists by AxMedian. Let us turn our attention to the coordinate system of observer $h$ illustrated by the right-hand side of Fig.4. Let $B \in w l_{h}(b)$ and $C \in w l_{h}(c)$ be distinct points such that $B_{\tau}=C_{\tau}$. Let $D$ be the center of masses of $b$ and $c$ at time instance $B_{\tau}=C_{\tau}$ according to observer $h$, i.e., $D:=c e n_{h}\left(b, c, B_{\tau}\right)$. Since the speeds and the rest masses of $b$ and $c$ coincide, their relativistic masses coincide by AxSpeed. But then, by definition of center of masses, $D$ is the midpoint of segment $[B C]$, i.e., $|B D|=|D C|$.

$w:=w_{h k}$ is a bijection taking lines to lines by Prop.3.1. Furthermore, it takes the midpoint of a segment to the midpoint of the $w$-image of 
the segment, i.e., $w((p+q) / 2)=(w(p)+w(q)) / 2$ for every $p, q \in \mathrm{Q}^{d}$. The proof of this statement is illustrated in Fig.5.

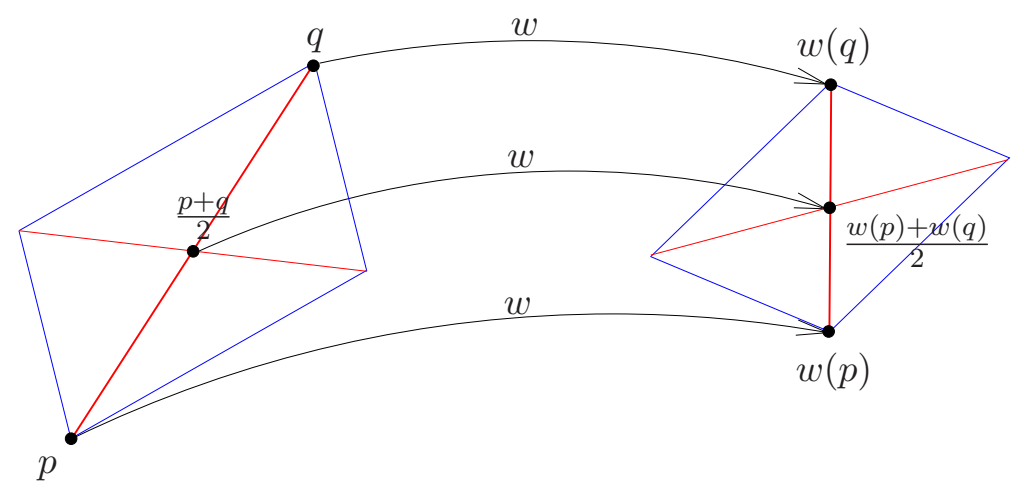

FiguRE 5. $w$ takes midpoint of a segment to the midpoint of the $w$-image of the segment. The proof of that is based on the fact that $w$ takes parallelograms to parallelograms and the diagonals of parallelograms bisect each other.

Now let us turn our attention to the coordinate system of observer $k$ illustrated by the left-hand side of Fig.4. Let $B^{\prime}, C^{\prime}$ and $D^{\prime}$ be the $w$-images of $B, C$ and $D$. Then $B_{\tau}^{\prime}=C_{\tau}^{\prime}=D_{\tau}^{\prime}$ by AxAbsSim. Furthermore, $D^{\prime}$ is the midpoint of segment $\left[B^{\prime} C^{\prime}\right]$, i.e., $\left|B^{\prime} D^{\prime}\right|=\left|D^{\prime} C^{\prime}\right|$ since $D$ is the midpoint of segment $[B C]$ and $w$ takes midpoints to midpoints. Furthermore, $D^{\prime} \in c e n_{k}(b, c)$, by $\mathrm{AxCen}{ }^{-}$, since $D \in c e n_{h}(b, c)$. Thus $D^{\prime}$ is the center of masses of $b$ and $c$ at time instance $B_{\tau}^{\prime}=C_{\tau}^{\prime}=D_{\tau}^{\prime}$ according to observer $k$, i.e., $D^{\prime}=\operatorname{cen}_{k}\left(b, c, B_{\tau}^{\prime}\right)$. But then, by definition of center of masses, $m_{k}(b)=m_{k}(c)=m_{0}(c)=m_{0}(b)$ since $\left|B^{\prime} D^{\prime}\right|=\left|D^{\prime} C^{\prime}\right|$. That completes the proof.

Proof of Thm.4.4. Assume first Kin $\cup\{$ AxRest $\}$. Let $k, h \in$ IOb. Let $w:=w_{k h}$. Then $w$ is a bijection taking lines to lines by Prop.3.1. Thus 
it takes parallel lines to parallel ones. Furthermore, it takes the midpoint of a segment to the midpoint of the $w$-image of the segment, i.e., $w((p+q) / 2)=(w(p)+w(q)) / 2$ for every $p, q \in \mathrm{Q}^{d}$. The proof of this statement is illustrated in Fig.5.

To prove that the world-view transformations are affine transformations in models of NewtDyn and SpecRelDyn, it is enough to prove that $|A C| /|C B|=|w(A) w(C)| /|w(C) w(B)|$ for every distinct $A, B, C \in \mathrm{Q}^{d}$ with $A_{\tau}=B_{\tau}=C_{\tau}$. To prove that, let $A, B$ and $C$ be distinct coordinate points such that $A_{\tau}=B_{\tau}=C_{\tau}$. We can assume that $C$ is between $A$ and $B$. See the left-hand side of Fig.6. Let $a \in$ IB

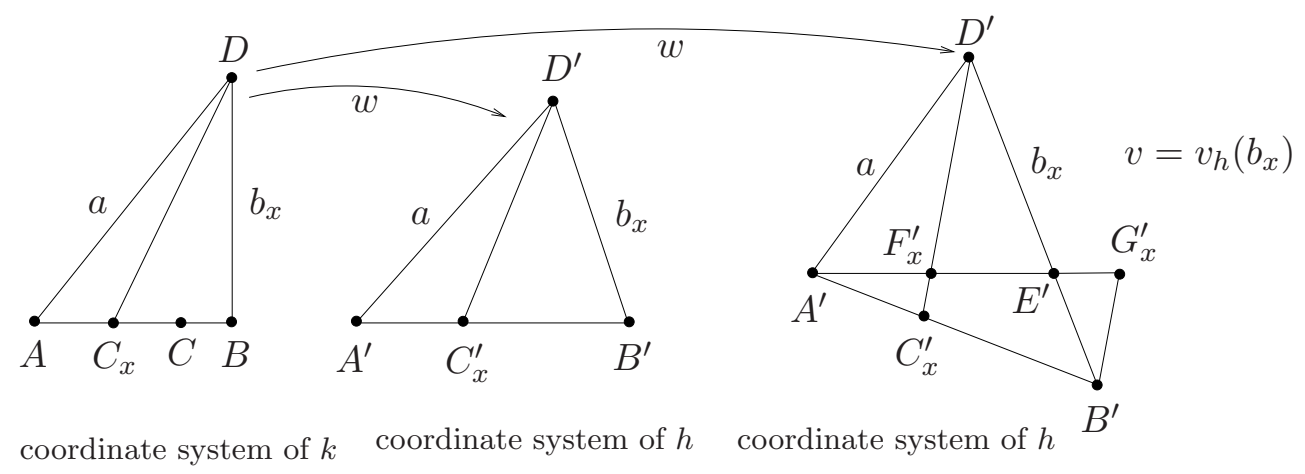

NewtDyn

SpecRelDyn

Figure 6. Illustration for the proof of Thm.4.4.

be such that $A \in w l_{k}(a)$ and $v_{k}(a) \neq 0$. Let $D \in w l_{k}(a)$ be such that $D_{\sigma}=B_{\sigma}$. For every $x \in \mathrm{Q}^{+}$, fix an inertial body $b_{x}$ such that $w l_{k}\left(b_{x}\right)=B D$ and the rest mass of $b$ is $x$, i.e., $m_{0}\left(b_{x}\right)=x$. Such bodies exist by AxRest. For every $x \in \mathrm{Q}^{+}$, let $C_{x}$ be the center of masses of $a$ and $b_{x}$ at time-instance $A_{\tau}=B_{\tau}$ according to $k$, i.e., $C_{x}:=\operatorname{cen}_{k}\left(a, b_{x}, A_{\tau}\right)$. By definition of the center of masses, 


$$
\begin{aligned}
\left|A C_{x}\right| /\left|C_{x} B\right|=m_{k}\left(b_{x}\right) / m_{k}(a) & =m_{0}\left(b_{x}\right) / m_{k}(a)=x / m_{k}(a) . \text { Let } \\
\lambda & :=\frac{1}{m_{k}(a)} .
\end{aligned}
$$

Then

$$
\forall x \in \mathrm{Q}^{+} \quad \frac{\left|A C_{x}\right|}{\left|C_{x} B\right|}=\lambda x .
$$

Let $A^{\prime}, B^{\prime}, D^{\prime}$ and $C_{x}^{\prime}$ be the $w$-images of $A, B, D$ and $C_{x}$, respectively.

Now assume NewtDyn. Then $m_{h}\left(b_{x}\right)=m_{0}\left(b_{x}\right)=x$ by Thm.4.3. Furthermore, $A_{\tau}^{\prime}=B_{\tau}^{\prime}=\left(C_{x}^{\prime}\right)_{\tau}$ by AxAbsSim, see the middle of Fig.6. Then $D^{\prime} C_{x}^{\prime}=\operatorname{cen}_{h}\left(a, b_{x}\right)$ by AxCen ${ }^{-}$. Thus $C_{x}^{\prime}$ is the center of masses of $a$ and $b_{x}$ at time instance $A_{\tau}^{\prime}$ according to $h$, i.e., $C_{x}^{\prime}=\operatorname{cen}_{h}\left(a, b_{x}, A_{\tau}^{\prime}\right)$. Thus $\left|A^{\prime} C_{x}^{\prime}\right| /\left|C_{x}^{\prime} B^{\prime}\right|=m_{h}\left(b_{x}\right) / m_{h}(a)=x / m_{h}(a)$. Let

$$
\lambda^{\prime}:=\frac{1}{m_{h}(a)} .
$$

Then

$$
\forall x \in \mathrm{Q}^{+} \quad \frac{\left|A^{\prime} C_{x}^{\prime}\right|}{\left|C_{x}^{\prime} B^{\prime}\right|}=\lambda^{\prime} x .
$$

Let us now consider the case $x=m_{k}(a)$. Then $C_{m_{k}(a)}$ is the midpoint of the segment $[A B]$, i.e., $\left|A C_{m_{k}(a)}\right|=\left|C_{m_{k}(a)} B\right|$ by (3) and (4). Then $C_{m_{k}(a)}^{\prime}$ is the midpoint of the segment $\left[A^{\prime} B^{\prime}\right]$, since $w$ takes the midpoint of a segment to the midpoint of the $w$-image of the segment. But then, by (4) and (6), $\lambda m_{k}(a)=\lambda^{\prime} m_{k}(a)=1$. Hence

$$
\lambda=\lambda^{\prime}
$$

Thus, by (4) and (6), $\forall x \in \mathrm{Q}^{+}\left|A C_{x}\right| /\left|C_{x} B\right|=\left|A^{\prime} C_{x}^{\prime}\right| /\left|C_{x}^{\prime} B^{\prime}\right|$. Clearly, there is an $x$ such that $C_{x}=C$. Then $|A C| /|C B|=\left|A^{\prime} C^{\prime}\right| /\left|C^{\prime} B^{\prime}\right|$, which completes the proof for the case of NewtDyn.

Now assume SpecReIDyn. See the right-hand side of Fig.6. Let us note, that the speed $v_{h}\left(b_{x}\right)$ is independent of the choice of $x$. Let $v$ denote this speed. Then $m_{h}\left(b_{x}\right)=m_{0}\left(b_{x}\right) / \sqrt{1-v^{2}}=x / \sqrt{1-v^{2}}$ by Thm.4.2. Clearly, $D^{\prime} C_{x}^{\prime}=\operatorname{cen}_{h}\left(a, b_{x}\right)$ by AxCen ${ }^{-}$. Thus $A_{\tau}^{\prime} \neq D_{\tau}^{\prime} \neq$ 
$B_{\tau}^{\prime}$. Let $E^{\prime} \in D^{\prime} B^{\prime}$ be such that $E_{\tau}^{\prime}=A_{\tau}^{\prime}$. Let $F_{x}^{\prime}$ be the intersection of $D^{\prime} C_{x}^{\prime}$ and $A^{\prime} E^{\prime}$. Clearly, $F_{x}^{\prime}=\operatorname{cen}_{h}\left(a, b_{x}, A_{\tau}^{\prime}\right)$. Thus

$$
\frac{\left|A^{\prime} F_{x}^{\prime}\right|}{\left|F_{x}^{\prime} E^{\prime}\right|}=\frac{m_{h}\left(b_{x}\right)}{m_{h}(a)}=\frac{x}{m_{h}(a) \sqrt{1-v^{2}}} .
$$

Let $G_{x}^{\prime} \in A^{\prime} E^{\prime}$ be such that $B^{\prime} G_{x}^{\prime}$ is parallel to $D^{\prime} C_{x}^{\prime}$. Now,

$$
\frac{\left|A^{\prime} C_{x}^{\prime}\right|}{\left|C_{x}^{\prime} B^{\prime}\right|}=\frac{\left|A^{\prime} F_{x}^{\prime}\right|}{\left|F_{x}^{\prime} G_{x}^{\prime}\right|}=\frac{\left|A^{\prime} F_{x}^{\prime}\right|}{\left|F_{x}^{\prime} E^{\prime}\right|} \frac{\left|F_{x}^{\prime} E^{\prime}\right|}{\left|F_{x}^{\prime} G_{x}^{\prime}\right|}=\frac{x}{m_{h}(a) \sqrt{1-v^{2}}} \frac{\left|D^{\prime} E^{\prime}\right|}{\left|D^{\prime} B^{\prime}\right|} .
$$

Let

$$
\lambda^{\prime \prime}:=\frac{\left|D^{\prime} E^{\prime}\right|}{m_{h}(a) \sqrt{1-v^{2}}\left|D^{\prime} B^{\prime}\right|} .
$$

Then

$$
\forall x \in \mathrm{Q}^{+} \quad \frac{\left|A^{\prime} C_{x}^{\prime}\right|}{\left|C_{x}^{\prime} B^{\prime}\right|}=\lambda^{\prime \prime} x .
$$

Now,

$$
\lambda=\lambda^{\prime \prime}
$$

can be proved by (3), (4) and (9) exactly the same way as $\lambda=\lambda^{\prime}$ was proved for the case of NewtDyn. The rest of the proof is analogous to the proof for the case of NewtDyn.

Proof of Thm.4.5. Let $a$ be an inertial body and $k, h \in \mathrm{IOb}$ be such that $v_{k}(a)<1$. Then, by Thm.3.5, $v_{h}(a)<1$ if $d \geq 3$. To prove that $v_{h}(a)<1$ for arbitrary $d$, let $b_{k}$ and $b_{h}$ be inertial bodies having rest masses such that $v_{k}\left(b_{k}\right)=0$ and $v_{h}\left(b_{h}\right)=0$. Such bodies exist by AxRest. By AxMedian, there is an observer according to which $b_{k}$ and $b_{h}$ have the same speeds. But then it can be proved that $v_{h}\left(b_{k}\right)<1$, cf. [5, Thm.2.7.2, p.110]. By that, it is easy to prove that $v_{h}(a)<1$, too.

Assume first, that $v_{k}(a) \neq 0$. We will use the proof of Thm.4.4 for the case of SpecRelDyn. So we can assume that $k, h$ and $a$ are as in the second paragraph of that proof, and let $A, B, b_{x}$ etc. be as in that 
proof, see the left-hand and right-hand sides of Fig.6. It can be proved that

$$
\frac{\mu\left(D^{\prime}, A^{\prime}\right)}{\mu\left(D^{\prime}, B^{\prime}\right)}=\frac{\mu(D, A)}{\mu(D, B)}=\sqrt{1-v_{k}(a)^{2}}
$$

by Thm.3.7 and Thm.4.4. Then

$$
\frac{\left|D^{\prime} E^{\prime}\right|}{\left|D^{\prime} B^{\prime}\right|}=\frac{\mu\left(D^{\prime}, E^{\prime}\right)}{\mu\left(D^{\prime}, B^{\prime}\right)}=\frac{\mu\left(D^{\prime}, E^{\prime}\right)}{\mu\left(D^{\prime}, A^{\prime}\right)} \frac{\mu\left(D^{\prime}, A^{\prime}\right)}{\mu\left(D^{\prime}, B^{\prime}\right)}=\frac{\sqrt{1-v^{2}} \sqrt{1-v_{k}(a)^{2}}}{\sqrt{1-v_{h}(a)^{2}}} .
$$

By this equation, (3), (8) and (10), we conclude that $m_{k}(a) \sqrt{1-v_{k}(a)^{2}}=m_{h}(a) \sqrt{1-v_{h}(a)^{2}}$.

Now assume that $v_{k}(a)=0$. Let $a^{*}$ be an inertial body such that $w l_{k}\left(a^{*}\right)=w l_{k}(a)$ and $m_{0}\left(a^{*}\right)=m_{k}(a)$. Such an $a^{*}$ exists by AxRest. Then

$$
m_{k}(a)=m_{0}\left(a^{*}\right)=m_{h}\left(a^{*}\right) \sqrt{1-v_{h}(a)^{2}}
$$

by Thm.4.2. Let $b$ be an inertial body such that $v_{k}(b) \neq 0$ and $w l_{k}(a) \cap w l_{k}(b) \neq \emptyset$. Clearly, $\operatorname{cen}_{k}(a, b)=\operatorname{cen}_{k}\left(a^{*}, b\right)$. Then $c e n_{h}(a, b)=$ $\operatorname{cen}_{h}\left(a^{*}, b\right)$ by $\mathrm{AxCen}{ }^{-}$. Thus $m_{h}(a)=m_{h}\left(a^{*}\right)$. This equation together with (11) completes the proof.

Proof of Thm.4.7. The proof is analogous to that of Thm.4.5. Let $a$ be an inertial body and $k, h \in \mathrm{IOb}$ be such that $v_{k}(a)$ is defined.

Assume first, that $v_{k}(a) \neq 0$. We will use the proof of Thm.4.4 for the case of NewtDyn. We can assume that $k, h$ and $a$ are as in that proof. By (3), (5) and (7) we get that $m_{k}(a)=m_{h}(a)$.

Now assume that $v_{k}(a)=0$. Let $a^{*}$ be an inertial body such that $w l_{k}\left(a^{*}\right)=w l_{k}(a)$ and $m_{0}\left(a^{*}\right)=m_{k}(a)$. Such an $a^{*}$ exists by AxRest. Then

$$
m_{k}(a)=m_{0}\left(a^{*}\right)=m_{h}\left(a^{*}\right)
$$

by Thm.4.3. Let $b$ be an inertial body such that $v_{k}(b) \neq 0$ and $w l_{k}(a) \cap w l_{k}(b) \neq \emptyset$. Clearly, $\operatorname{cen}_{k}(a, b)=\operatorname{cen}_{k}\left(a^{*}, b\right)$. Then $c e n_{h}(a, b)=$ 
$\operatorname{cen}_{h}\left(a^{*}, b\right)$ by $\mathrm{AxCen}{ }^{-}$. Thus $m_{h}(a)=m_{h}\left(a^{*}\right)$. This equation together with (12) completes the proof.

Proof of Cor.4.8. Assume NewtDyn. Let $k, h \in \mathrm{IOb}$ and $a, b \in \mathrm{IB}$. We would like to prove that

$$
w_{k h}\left[\operatorname{cen}_{k}(a, b)\right]=\operatorname{cen}_{h}(a, b) .
$$

First assume that $v_{k}(a)$ is "infinite," i.e., undefined. Then $v_{h}(a)$ is also "infinite," by AxAbsSim. Hence, $\operatorname{cen}_{k}(a, b)=\operatorname{cen}_{h}(a, b)=\emptyset$. Then (13) holds. The same holds if $v_{k}(b)$ is "infinite".

If $w l_{k}(a) \cap w l_{k}(b) \neq \emptyset$, (13) holds by $\mathrm{AxCen}{ }^{-}$. Thus we can assume that $w l_{k}(a) \cap w l_{k}(b)=\emptyset$. Now assume that $v_{k}(a)$ and $v_{k}(b)$ are not "infinite," i.e., both are defined. Then $v_{h}(a)$ and $v_{h}(b)$ are also defined by AxAbsSim. First we prove that $w_{k h}\left[\operatorname{cen}_{k}(a, b)\right] \subseteq c e n_{h}(a, b)$. To prove this statement, let $C \in \operatorname{cen}_{k}(a, b)$. Let $A \in w l_{k}(a)$ and $B \in$ $w l_{k}(b)$ be such that $A_{\tau}=B_{\tau}=C_{\tau}$. Then, by definition of center of masses, $|A C| /|C B|=m_{k}(b) / m_{k}(a)$. Let $A^{\prime}, B^{\prime}$ and $C^{\prime}$ be the $w_{k h^{-}}$ images of $A, B$ and $C$. Clearly, $A_{\tau}^{\prime}=B_{\tau}^{\prime}=C_{\tau}^{\prime}$ by AxAbsSim. By Thm.4.7, $m_{k}(b)=m_{h}(b)$ and $m_{k}(a)=m_{h}(a)$. By Thm.4.4, $w_{k h}$ is a bijective affine transformation. Consequently,

$$
\left|A^{\prime} C^{\prime}\right| /\left|C^{\prime} B^{\prime}\right|=|A C| /|C B|=m_{k}(b) / m_{k}(a)=m_{h}(b) / m_{h}(a) .
$$

Hence $C^{\prime} \in \operatorname{cen}_{h}(a, b)$. Thus $w_{k h}\left[\operatorname{cen}_{k}(a, b)\right] \subseteq \operatorname{cen}_{h}(a, b)$. Analogously, $w_{h k}\left[\operatorname{cen}_{h}(a, b)\right] \subseteq \operatorname{cen}_{k}(a, b)$ holds. Since $w_{k h}$ and $w_{h k}$ are bijections and inverses of each other, we conclude that (13) holds.

\section{Concluding Remarks}

We have shown that Newtonian and relativistic dynamics can be axiomatized (within FOL) such that they differ in one axiom only. However, in the level of consequences, they have radical differences. 
The most surprising difference is that $A x C e n$, an apparently harmless consequence of Newtonian dynamics, is inconsistent with relativistic dynamics.

\section{Notes}

${ }^{1}$ Replacing the field of real numbers by an ordered field not just increases the flexibility of our theories but makes it possible to keep them within FOL, which is crucial in axiomatic foundations, see, e.g., [5, Appendix: Why first-order logic?].

${ }^{2}$ That is, a linearly ordered field in which positive elements have square roots.

\section{REFERENCES}

[1] H. Andréka, P. Burmeister, and I. Németi. Quasivarieties of partial algebras a unifying approach towards a two-valued model theory for partial algebras. Studia Sci. Math. Hungar. 16, 1981, pp.325-372.

[2] H. Andréka, J. X. Madarász, and I. Németi. "Logical analysis of special relativity theory," in: J. Gerbrandy, M. Marx, M. de Rijke, and Y. Venema (Eds.), Essays dedicated to Johan van Ben- them on the occasion of his 50 th birthday. Vossiuspers, Ams- terdam University Press, 1999. CD-ROM, ISBN: 905629 104 1, http://www.illc.uva.nl/j50.

[3] H. Andréka, J. X. Madarász, and I. Németi. "Logical axiomatizations of spacetime. Samples from the literature," in: Non-Euclidean geometries, volume 581 of Mathematics and Its Applications. New York: Springer, 2006, pp.155-185.

[4] H. Andréka, J. X. Madarász, and I. Németi, "Logic of space-time and relativity theory," in: M. Aiello, I. Pratt-Hartmann and J. van Benthem (Eds.), Handbook of Spatial Logics. Dordrecht: Springer, 2007, pp.607-711.

[5] H. Andréka, J. X. Madarász, and I. Németi, "On the logical structure of relativity theories." Research report, Alfréd Rényi Institute of Mathematics, Budapest, 2002. With contributions from A. Andai, G. Sági, I. Sain and Cs. Tőke. http://www.math-inst.hu/pub/algebraic-logic/Contents.html. 1312 pp.

[6] H. Andréka, J. X. Madarász, I. Németi, and G. Székely, "Axiomatizing relativistic dynamics without conservation postulates," in: Studia Logica 89, 2, 2008, pp.163-186. 
[7] J. Ax, "The elementary foundations of spacetime," in: Foundations of Physics 8, 7-8, 1978, pp.507-546.

[8] S. A. Basri, A Deductive Theory of Space and Time. Amsterdam: NorthHolland 1966.

[9] T. Benda, "A Formal Construction of the Spacetime Manifold," in: Journal of Philosophical Logic 37, 5, 2008, pp.441-478.

[10] C. C. Chang and H. J. Keisler, Model theory. Amsterdam: North-Holland 1973, $1977,1990$.

[11] H. D. Ebbinghaus, J. Flum and W. Thomas, Mathematical logic. New York: Springer-Verlag 1994.

[12] R. Goldblatt, Orthogonality and spacetime geometry. New York: SpringerVerlag 1987.

[13] J. X. Madarász. Logic and Relativity (in the light of definability theory). PhD thesis, Eötvös Loránd Univ., Budapest, 2002.

[14] J. X. Madarász, I. Németi, and G. Székely, "Twin paradox and the logical foundation of relativity theory," in: Foundations of Physics 36, 5, 2006, pp.681714 .

[15] J. X. Madarász, I. Németi, and G. Székely, "First-order logic foundation of relativity theories," D. Gabbay, S. Goncharov and M. Zakharyaschev (Eds.), in: Mathematical problems from applied logic II. New York: Springer, 2007, pp.217-252.

[16] J. X. Madarász, I. Németi, and Cs. Tőke. On generalizing the logic-approach to space-time towards general relativity: first steps. in: V. F. Hendricks, F. Neuhaus, S. A Pedersen, U. Scheffler and H. Wansing (Eds.), First-Order Logic Revisited, Logos Verlag, Berlin, 2004, pp.225-268.

[17] V. Pambuccian, "Alexandrov-Zeeman type theorems expressed in terms of definability," in: Aequationes Mathematicae 74, 3, 2007, pp.249-261.

[18] G. Székely, First-Order Logic Investigation of Relativity Theory with an Emphasis on Accelerated Observers. PhD thesis, Eötvös Loránd Univ., Budapest, 2009, submitted.

[19] J. Väänänen, "Second-order logic and foundations of mathematics," in: Bulletin of Symbolic Logic 7, 4, 2001, pp.504-520. 
[20] J. Woleński "First-order logic: (philosophical) pro and contra," in: V. F. Hendricks, F. Neuhaus, S. A. Pedersen, U. Scheffler and H. Wansing (Eds.), FirstOrder Logic Revisited. Berlin: Logos Verlag 2004, pp.369-398. 